\title{
Characteristics of a Cutoff Cavity Probe Applicable to Crack Detection Using the Forced Resonance Microwave Method
}

\author{
Ki-Chai Kim ${ }^{1, *} \cdot$ Jong-Woo Kim² $\cdot$ Jae-Yong Kwon ${ }^{3} \cdot \mathrm{No}^{2}$ Weon Kang ${ }^{3}$
}

\begin{abstract}
This paper presents the current characteristics inside a cutoff cavity slot probe applied to crack detection using the forced resonance microwave method (FRMM). Crack detection using FRMM has two stages: preparation and detection. In the preparation stage, the current characteristics inside the probe with a shorting plate are important for determining the crack signal and detection sensitivity. The cutoff cavity probe produces a forced resonance by adjusting a control element. There are two kinds of forced resonance: series resonance (SR) and parallel resonance (PR). Four types of current characteristics are applied to crack detection using FRMM: SR, the region around SR, the region around $\mathrm{PR}$, and non-resonance. These current characteristics are discussed from the point of view of current change for crack detection. The experimental results are compared with the theoretical results to check the current state inside the cutoff cavity probe.
\end{abstract}

Key Words: Crack Detection, Cutoff Cavity, Forced Resonance, Microwave Method, Nondestructive Testing.

\section{INTRODUCTION}

Several nondestructive testing (NDT) techniques can be used to detect surface cracks in metal materials. These techniques include eddy current testing, AC (alternating current) field measurement, acoustic emission testing, and ultrasonic testing [1-3]. Cracks in metal that are concealed by paint, composite laminates, corrosion protection substances, and rust can be detected easily using microwave techniques compared with other techniques. Studies have shown that nondestructive surface crack detection at the microwave and mm-wave frequencies is capable of detecting and sizing surface cracks in metals [4-9]. In
[4], an open-ended waveguide operating at $20 \mathrm{GHz}$ was utilized as a sensor to detect smaller surface cracks, such as fatigue cracks from changes in the reflection coefficient. However, it can easily be affected by noise, air gap, and other factors [5]. To resolve this problem, several critical parameters of the open-ended waveguide technique have been investigated $[6,7]$. To obtain a higher spatial resolution, the open-ended waveguide technique needs to be used at higher frequencies [8]. Alternatively, open-ended coaxial probes have also been examined [9]. Recently, resonant probes have been reported to design near-field sensors for crack detection in metallic materials [10]. Resonant probes provide many advantages compared with other probes based on measure-

Manuscript received March 13, 2020 ; Revised June 24, 2020 ; Accepted July 27, 2020. (ID No. 20200313-031J)

${ }^{1}$ Department of Electrical Engineering, Yeungnam University, Gyeongsan, Korea.

${ }^{2}$ EMC Team, Korea Marine Equipment Research Institute, Busan, Korea

${ }^{3}$ Center for Electromagnetic Metrology, Korea Research Institute of Standards and Science (KRISS), Daejeon, Korea.

"Corresponding Author: Ki-Chai Kim (e-mail: kckim@ynu.ac.kr)

This is an Open-Access article distributed under the terms of the Creative Commons Attribution Non-Commercial License (http://creativecommons.org/licenses/by-nc/4.0) which permits unrestricted non-commercial use, distribution, and reproduction in any medium, provided the original work is properly cited.

(c) Copyright The Korean Institute of Electromagnetic Engineering and Science. All Rights Reserved. 
ing changes in the reflection coefficient. Several technologies with resonant probes for crack detection have been reported, including a microstrip linear resonator, a quarter-wavelength microstrip line, a dual-behavior resonator filter, and a complementary split-ring resonator [11-14]. A more general review of state-of-the-art NDT techniques using microwave technologies is described in [15]. These techniques require a vector network analyzer (VNA), which makes the application of these types of probes difficult from a practical standpoint.

Conversely, cutoff cavity probes using forced resonance have been utilized for crack detection in metallic materials [16]. This method is called the forced resonance microwave method (FRMM). The advantage of this method is that VNA is not required, and thus the crack detection system can be established at low cost. FRMM consists of two stages: preparation and detection. It uses the current deviation from the resonance state for the cutoff cavity probe without a crack (corresponding to the preparation stage) and with a crack (corresponding to the detection stage). Therefore, knowledge of the current characteristics and the forced resonance modes of the cutoff-cavity probe in the preparation stage is needed as a reference to obtain the crack signals.

This paper presents the current states of the forced resonance of the cutoff cavity applied to crack detection using FRMM. The cutoff-cavity probe produces forced resonance by adjusting a control element. There are two kinds of forced resonance: series resonance (SR) and parallel resonance (PR). The SR causes a maximum current, and the $\mathrm{PR}$ and the region surrounding it produce sluggishly gradient currents. The steepest gradient currents occur around the SR. The minimum current occurs at non-resonance (NR) using the control element. Therefore, there are four types of current states in FRMM: maximum current (at the SR), minimum current (at the NR), rapidly changing current (around the SR), and slowly changing current (at the PR and around it). These current characteristics have been classified. The FRMM mainly uses SR, but PR and NR can also be used for crack detection. However, PR and NR are not as advantageous as SR. To verify the current state inside the cutoff cavity, the experimental results are compared with the theoretical results.

\section{SYSTEM DESCRIPTION}

The geometry of the surface crack detection system is shown in Fig. 1. The detection system consists of four parts: (1) the cutoff cavity-backed narrow slot probe with internal posts \#1 and \#2, (2) the external control element $j X_{1}$ for cavity resonance control, (3) the signal generator, and (4) the detector for reading the current states on post \#2 and the crack signals. A voltmeter or an ammeter can be used as a detector. In Fig. 1(a),
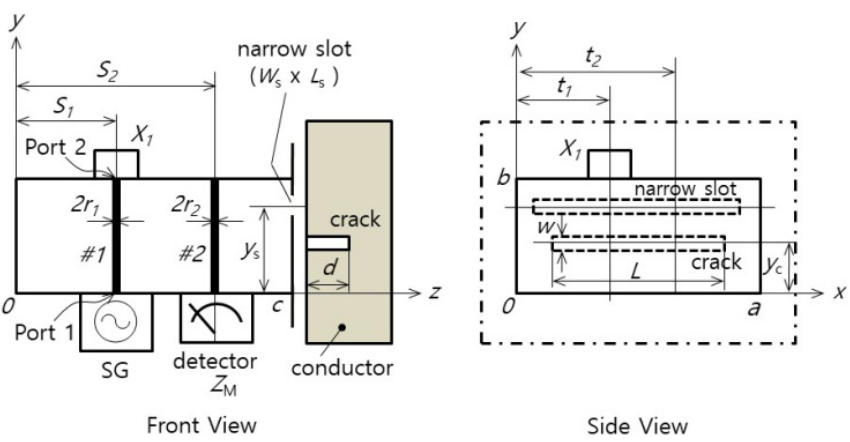

(a)
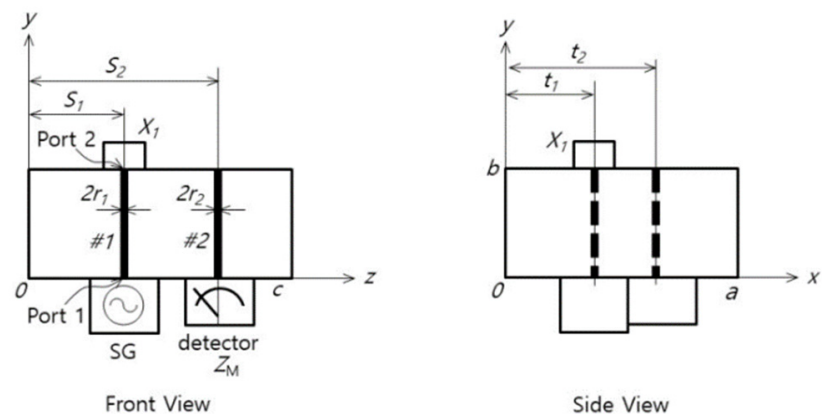

(b)

Fig. 1. A cutoff cavity-backed slot probe for crack detection. (a) In the detection stage, the cavity probe and metal are in perfect contact and scanned on the metal surface. (b) In the preparation stage, the probe is enclosed by a shorting plate.

the probe and metal are presented separately, but in actual detection, they are in perfect contact. In the preparation stage, the slot is short, and the probe becomes a perfect cavity, as shown in Fig. 1(b).

The probe dimensions are chosen such that the cross-section $a \times b$ of the cavity corresponds to the cutoff condition for the waveguide of the same cross-section when the cavity is empty. For this reason, it is called a cutoff cavity. In the probe, a feed post (\#1) of radius $r_{1}$ is located at $x=t_{1}$ and $z=s_{1}$. The external control element $j X_{1}$ acts as a reactance element and is connected to the top of the feed post. A detector is connected to the bottom of the parasitic post (\#2) of radius $r_{2}$ at $x=t_{2}$ and $z=s_{2}$. The detector is used to check the desired forced resonance or non-resonance currents and to measure the output crack signals. Each parameter referenced in Fig. 1 is listed in Table 1.

To obtain the forced resonance and non-resonance current characteristics, Galerkin's method of moments (MoM) is used to solve the integral equations for the current distributions on the two posts inside the probe. For the evaluation of the forced resonance characteristics, a two-port network representation is used with admittance parameters to derive the deterministic equation for the length of the control element, as described in Section III-2. 
Table 1. Geometric parameters

\begin{tabular}{lc}
\hline \multicolumn{1}{c}{ Parameter } & Value $(\mathrm{mm})$ \\
\hline Cavity width, $a$ & 40 \\
Cavity height, $b$ & 20 \\
Cavity depth, $c$ & 80 \\
$x$ position of the feed post \#1, $t_{1}$ & 20 \\
$x$ position of the parasitic post \#2, $t_{2}$ & 20 \\
$z$ position of the feed post \#1, $S_{1}$ & 30 \\
$z$ position of the parasitic post \#2, $S_{2}$ & 60 \\
Radius of the feed post \#1, $r_{1}$ & 1 \\
Radius of the parasitic post \#2, $r_{2}$ & 1 \\
Slot center position, $y_{s}$ & 10 \\
Crack center position, $y_{c}$ & - \\
Slot width, $W_{s}$ & 5 \\
Slot length, $L_{s}$ & 40 \\
Crack width, $W$ & 1 \\
Crack length, $L$ & 40 \\
Crack depth, $d$ & 1 \\
External reactance, $X_{1}$ & Variable \\
Detector impedance, $Z_{M}$ & $5 \mathrm{k} \Omega$ \\
\hline
\end{tabular}

\section{THEORETICAL ANALYSIS OF FRMM}

\section{Integral Equations}

As shown in Fig. 1(b), for the preparation stage, the probe with a metal shorting plate in the absence of a crack resonates forcefully in the cutoff cavity using the control element $j X_{1}$ connected to the top of feed post \#1. The voltage $V_{1}$ is applied at $y=0, x=t_{1}$, and $z=s_{1}$. The detector is also connected to the bottom of parasitic post \#2 at $y=0, x=t_{2}$, and $z=s_{2}$.

If the cutoff cavity is fed by a delta gap generator as a voltage source, the integral equations for the unknown electric currents $\bar{J}_{1}$ and $\bar{J}_{2}$ on feed post \#1 and parasitic post \#2 can be respectively written as follows:

$$
\begin{array}{r}
\oiint_{S_{1}^{\prime}} \overline{\bar{K}}_{e e 11}^{\mathrm{I}} \cdot \bar{J}_{1} d S_{1}^{\prime}+\oiint_{S_{2}^{\prime}} \overline{\bar{K}}_{e e 12}^{\mathrm{I}} \cdot \bar{J}_{2} d S_{2}^{\prime} \\
=-\hat{y} V_{1} \delta(y)+\hat{y} j X_{1} I_{1}(b) \delta(y-b) \\
\oiint_{S_{1}^{\prime}} \overline{\bar{K}}_{e e 21}^{\mathrm{I}} \cdot \bar{J}_{1} d S_{1}^{\prime}+\oiint_{S_{2}^{\prime}} \overline{\bar{K}}_{e e 22}^{\mathrm{I}} \cdot \bar{J}_{2} d S_{2}^{\prime} \\
=-\hat{y} Z_{M} I_{2}(0) \delta(y)
\end{array}
$$

where $\overline{\bar{K}}_{e e i j}^{\mathrm{I}}(i, j=1$ or 2$)$ is the dyadic Green function in region I (inside the cavity), which yields the electric field due to the current source. Superscript I denotes cavity region I, and subscripts 1 and 2 represent feed post \#1 and parasitic post \#2, respectively. $\delta(*)$ is the Dirac delta function, and $\hat{y}$ is a unit vector in the $y$-direction. $I_{2}(0)$ is the current at the ammeter loading position, and $I_{1}(b)$ is the current at the loading positions of control element $j X_{1} . Z_{\mathrm{M}}$ is the impedance of the detector. For convenience and simplicity, the current $I_{2}(0)$ at the ammeter loading position is expressed as $I_{2 \mathrm{~L}}$.

To solve the simultaneous integral equations for the unknowns, $\bar{J}_{1}$ and $\bar{J}_{2}$ are expanded as follows:

$$
\begin{aligned}
& \bar{J}_{1}(y)=\hat{y} \sum_{k=0}^{K} a_{1 k} \cos \left(\frac{k \pi y}{b}\right) \\
& \bar{J}_{2}(y)=\hat{y} \sum_{l=0}^{L} a_{2 l} \cos \left(\frac{l \pi y}{b}\right)
\end{aligned}
$$

where $a_{1 k}$ and $a_{2 l}$ are complex expansion coefficients. The integral equations are solved by applying Galerkin's MoM. Once the current distributions on the internal posts are obtained, the forced resonance characteristics can be considered for the probe by adjusting the control element.

\section{Forced Resonance of Cutoff Cavity}

When the probe is short-circuited with a shorting plate, we can obtain the forced resonance characteristics by treating the cutoff cavity as a two-port network, with a voltage applied at port 1 and the control element at port 2, as shown in Fig. 1. The relations between the terminal voltages and currents at each port can be expressed using admittance parameters $y_{i j}(i, j=1,2)$ :

$$
\left(\begin{array}{l}
i_{1} \\
i_{2}
\end{array}\right)=\left(\begin{array}{cc}
y_{11} & -y_{12} \\
-y_{21} & y_{22}
\end{array}\right)\left(\begin{array}{l}
v_{1} \\
v_{2}
\end{array}\right) .
$$

The terminal voltage at port 2 can be expressed as $i_{2}=$ $-\left(1 / j X_{1}\right) v_{2}=-Y_{1} v_{2}$, as a reactance element is connected to port 2 . Therefore, by putting this relationship in (5), we can obtain the input impedance at the driving point in the following form:

$$
Z_{\text {in }}=\frac{\left(y_{22}+Y_{1}\right)}{y_{11}\left(y_{22}+Y_{1}\right)-y_{12}^{2}} .
$$

If the above impedance expression is used, the resonant condition of the cutoff cavity is

$$
\operatorname{Im}\left\{Z_{i n}\left(y_{i j}, j X_{1}\right)\right\}=0 .
$$

The short-circuited transmission line with the characteristic impedance $Z_{0}$ and length $h_{1}$ constitutes the control element (reactance element). Therefore, the expression for the reactance is $j X_{1}=j Z_{0} \tan \left(k h_{1}\right)$, where $k$ is the propagation constant of the two parallel wires.

The reactance of the control element that forcibly resonates the cutoff cavity can be obtained from (7). Thus, the length of the reactance that resonates with the cutoff cavity can be expressed as:

$$
k h_{1}=\tan ^{-1}\left\{\frac{2 y_{11}^{I}}{z_{0}\left(-F \pm \sqrt{E^{2}-4 y_{11}^{I} H}\right)}\right\}
$$


where

$$
\begin{gathered}
F=y_{12}^{I} y_{21}^{I}-2 y_{11}^{I} y_{22}^{I}-y_{12}^{R} y_{21}^{R} \\
H=y_{22}^{I} y_{11}^{I} y_{22}^{I}+y_{22}^{I} y_{12}^{R} y_{21}^{R} \\
-y_{22}^{I} y_{12}^{I} y_{21}^{I}+y_{22}^{R} y_{11}^{I} y_{22}^{R}-y_{22}^{R} y_{12}^{I} y_{21}^{R}
\end{gathered}
$$

$y_{i j}^{R}$ and $y_{i j}^{I}$ are the real and imaginary parts of $y_{i j}$, respectively. In (8), the positive and negative signs in the denominator produce SR and PR, respectively.

\section{THEORETICAL RESULTS AND CLASSIFICATION}

\section{OF CURRENT STATES}

\section{Forced Resonance Characteristics}

The dimensions of the cutoff cavity probe used in the numeric calculation are $a=40 \mathrm{~mm}, b=20 \mathrm{~mm}, c=80 \mathrm{~mm}$, $s_{1}=30 \mathrm{~mm}, s_{2}=60 \mathrm{~mm}, t_{1}=t_{2}=a / 2$, and $r_{1}=r_{2}=$ $1 \mathrm{~mm}$. The operating frequencies are $1 \mathrm{GHz}$ and $2 \mathrm{GHz}$, and the dimension $a=40 \mathrm{~mm}$ (cutoff frequency $3.75 \mathrm{GHz}$ ) meets the cutoff conditions for these frequencies. An ammeter is used as a detector, but a voltmeter can also be used. The ammeter and a diode detect the crack signals rectified by the high-frequency diodes (1SS99; NEC Corp., Tokyo, Japan) connected to parasitic post \#2. Their impedance is $Z_{M}=5.23 \mathrm{k} \Omega[5 \mathrm{k} \Omega$ (ammeter) $+230 \Omega$ (diode)].

The probe can resonate forcefully in SR or PR by adjusting the control element according to (8), and SR is used for crack detection [16]. This paper presents the current states of the forced resonance of the cutoff cavity for crack detection using FRMM.

Fig. 2 shows the frequency characteristics of the length of the control element, $j X_{1}=j Z_{0} \tan \left(k h_{1}\right)$, which occurs with forced resonance from (8) when the cutoff cavity probe resonates forcefully from adjusting the control element for the ammeter $\left(Z_{M}=5.23 \mathrm{k} \Omega\right)$ connection. For the ammeter connection at $1 \mathrm{GHz}, \mathrm{SR}$ and PR occur at $k h_{1}=1.936\left(X_{1}=\right.$ $-130.77 \Omega$ )and $k h_{1}=0.971\left(X_{1}=73.18 \Omega\right)$, respectively. At $2 \mathrm{GHz}, \mathrm{SR}$ occurs at $k h_{1}=1.411\left(X_{1}=310.71 \Omega\right)$, and PR occurs at $k h_{1}=2.134\left(X_{1}=-79.17 \Omega\right)$. These resonance modes are summarized in Table 2.

As shown in Fig. 2, the cutoff cavity probe generates SR or $\mathrm{PR}$ at given frequencies by adjusting the control element $j X_{1}$ for the ammeter connection. The PR for the ammeter connection occurs at $k h_{1} \approx 0\left(X_{1} \approx 0 \Omega\right)$ at around $1.60 \mathrm{GHz}$, as shown in Fig. 2. The frequency of $1.60 \mathrm{GHz}$ is critical for determining the resonance. The vicinity of $1.60 \mathrm{GHz}$ is sensitive when obtaining the resonance by adjusting the control element and should not be in the crack detection using FRMM. The characteristics of a voltmeter connection are almost the same as those of the ammeter connection. As mentioned previously, the forced resonances other than those at $1.60 \mathrm{GHz}$ are useful for crack detection by the FRMM. It is necessary to analyze the

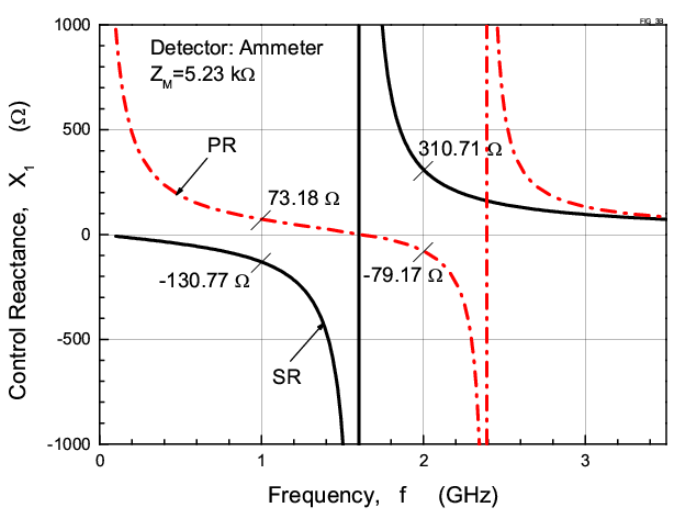

(a)

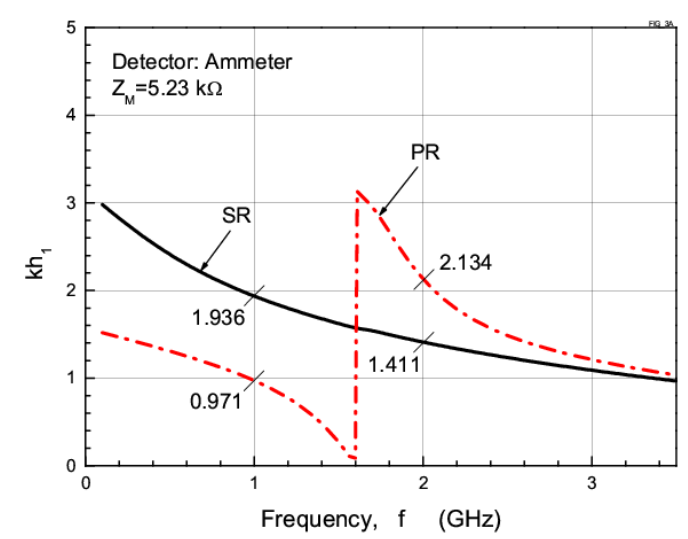

(b)

Fig. 2. Frequency characteristics of (a) the reactance and (b) length of the control element $\left(k h_{1}\right)$ for an ammeter as a detector.

Table 2. Resonance modes of the cutoff cavity probe

\begin{tabular}{cccc}
\hline $\begin{array}{c}\text { Resonance } \\
\text { frequency }(\mathrm{GHz})\end{array}$ & $\begin{array}{c}\text { Type of } \\
\text { resonance }\end{array}$ & $\begin{array}{c}\text { External } \\
\text { reactance }(\Omega)\end{array}$ & $k h_{1}$ \\
\hline 1 & SR & -130.77 & 1.936 \\
& PR & 73.18 & 0.971 \\
2 & NR & 192.76 & 1.317 \\
& SR & 310.71 & 1.411 \\
& PR & -79.17 & 2.134 \\
& NR & 95.39 & 1.088 \\
\hline
\end{tabular}

current states of the cutoff cavity probe at the resonance and around it. Knowledge of the input impedance characteristic of the cutoff cavity and the detector current (the ammeter connection point current) is needed to check the types of resonance.

Fig. 3 shows the detector current and input reactance versus the length of the control reactance when the operating frequency is fixed at $1 \mathrm{GHz}$ and $2 \mathrm{GHz}$ as an example. To determine whether $\mathrm{SR}$ or $\mathrm{PR}$ is occurring, it also shows the input reactance of the cutoff cavity probe. As shown in Fig. 3(a), for the ammeter connection at $1 \mathrm{GHz}, \mathrm{SR}$ occurs when $k h_{1}=1.936$ $\left(X_{1}=-130.77 \Omega\right)$. The maximum current state also occurs. 


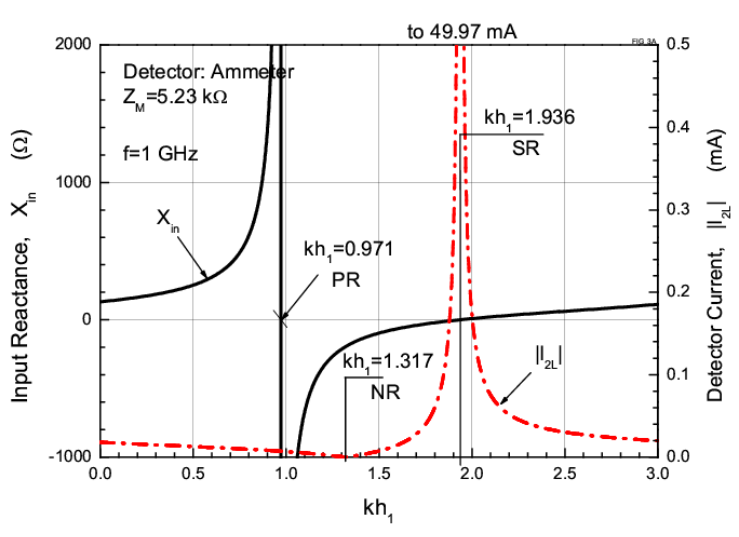

(a)

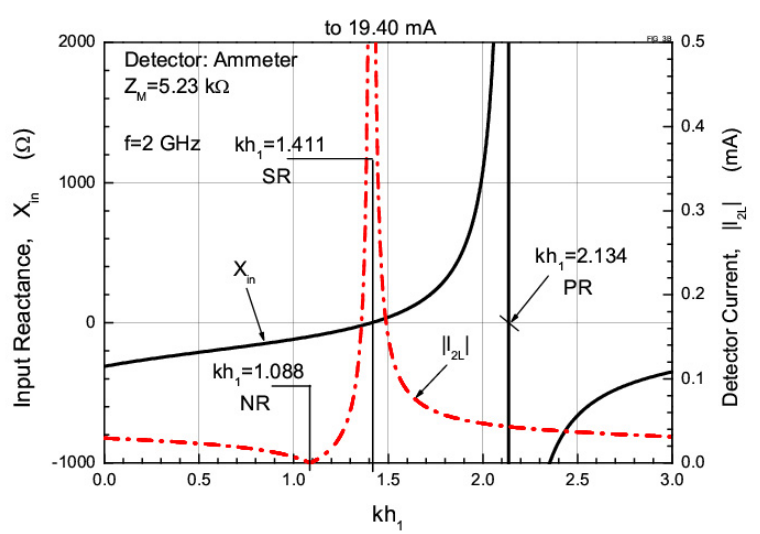

(b)

Fig. 3. Load current and input reactance of the cutoff cavity probe versus control element length: (a) $1 \mathrm{GHz}$ and (b) $2 \mathrm{GHz}$.

PR occurs when $k h_{1}=0.971\left(X_{1}=73.18 \Omega\right)$, and the current state slowly changes. The minimum current $(\approx 0 \mathrm{~mA})$ appears when $k h_{1}=1.317\left(X_{1}=192.76 \Omega\right)$, which is the NR state. For the ammeter connection at $2 \mathrm{GHz}$ shown in Fig. 3(b), SR occurs when $k h_{1}=1.411\left(X_{1}=310.71 \Omega\right)$, and the input reactance, $X_{\text {in }}$, changes from capacitive to inductive. The maximum current state occurs. PR occurs when $k h_{1}=$ $2.134\left(X_{1}=-79.17 \Omega\right)$, and the input reactance changes from inductive to capacitive. The current state slowly changes. The detector current also appears to be minimized $(\approx 0 \mathrm{~mA})$ at $k h_{1}=1.088\left(X_{1}=95.39 \Omega\right)$, which is the NR state.

\section{Classification of Current States}

Based on the current states for the ammeter connection in Fig. 3, the current states of the cutoff cavity probe have a maximum current (at SR), minimum current (at NR), rapidly changing current (around SR), and slowly changing current (at PR and around it). These detector current states and resonant types are illustrated in Fig. 4. There are four kinds of current states: (1) SR with maximum current, (2) PR and the surrounding region, which has a slowly changing current, (3) NR with minimum current, and (4) region around the SR with a rapidly changing

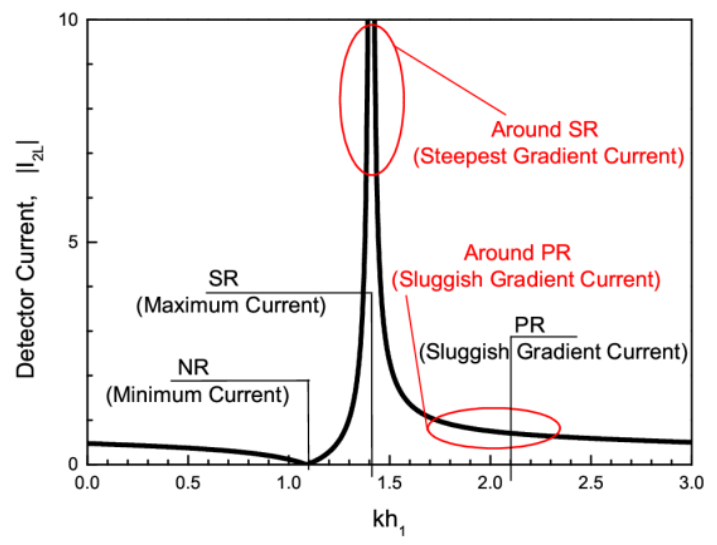

Fig. 4. Current configuration.

current. These current states are applicable to crack detection using FRMM. One of the four current states can be used for more sensitive crack detection when setting the reference current in the preparation stage. Crack signals are detected from the deviations of the reference current.

Fig. 5 shows the classification of resonant types and directions of the detector needle movement as the crack signal appears with the four current states in the crack detection stage. Fig. 5(a) shows the three types of resonance: (1) forced resonance (SR and PR), (2) NR, and (3) region around the forced resonance. As mentioned previously, SR has the maximum current, NR has the minimum current, $\mathrm{PR}$ and the surrounding region have a slowly changing current, and the region around the SR has a rapidly changing current, as shown in Fig. 4. For these current states, the directions of the needle movement are shown in Fig. 5(b). The needle moves in the lower or upper direction depending on the state of crack at a given frequency. The needle movement direction should be discussed in detail at the crack detection stage. However, this paper presents only one example. The needle movement directions for the crack of $W=1 \mathrm{~mm}$ and $d=1 \mathrm{~mm}$ are shown in Fig. 5(b). In this case, when the probe scans a crack in the detection stage, the needle moves in the lower directional movement if we use SR (maximum current) and NR (minimum current). For PR (slowly changing current), the needle moves in the upper direction. Using the region around SR (rapidly changing current) and around PR (slowly changing current), the needle also moves in the upper directions when the probe scans a crack. To check the needle movement shown in Fig. 5(b), the experimental results for the crack of $W=$ $1 \mathrm{~mm}$ and $d=1 \mathrm{~mm}$ at $1 \mathrm{GHz}$ are shown in Table 3. As a future study on the detection stage, more detailed research on the movement direction of the needle for various crack dimensions is needed.

As a result, using SR and the region around SR leads to the best sensitivity for crack detection, as the current changes have the steepest gradient. PR and the region around the PR can be used for crack detection, but sensitivity is lower than that of the 


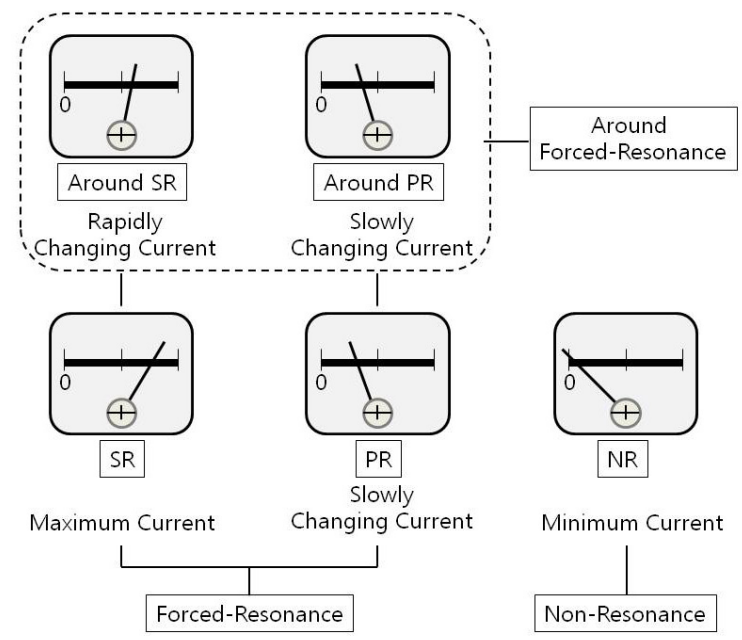

(a)

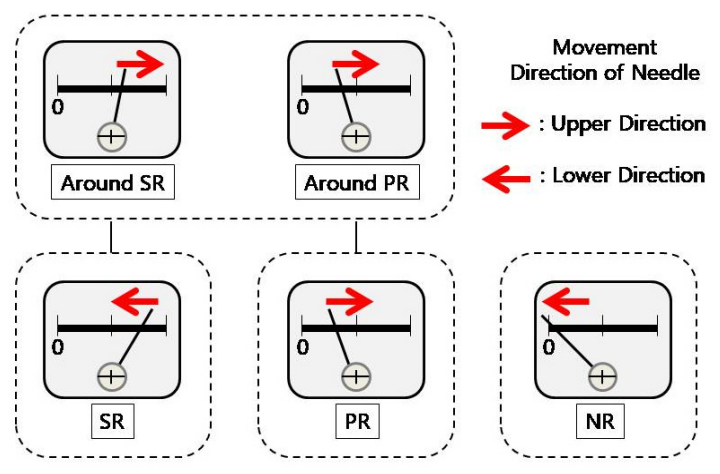

(b)

Fig. 5. Classification of (a) resonant types and (b) movement directions of a needle according to the current configuration.

Table 3. Experimental results of the movement direction of the needle

\begin{tabular}{lcccc}
\hline $\begin{array}{c}\text { Type of } \\
\text { resonance }\end{array}$ & $\begin{array}{c}\text { Reference } \\
\left|\mathrm{I}_{2 \mathrm{~L}}\right|(\mu \mathrm{A})\end{array}$ & $\begin{array}{c}\text { Crack } \\
\text { signal } \\
(\mu \mathrm{A})\end{array}$ & $k h_{1}$ & $\begin{array}{c}\text { Movement } \\
\text { direction of } \\
\text { the needle }\end{array}$ \\
\hline Around SR & 16.50 & 16.80 & 1.908 & $\rightarrow$ \\
SR & 17.20 & 16.90 & 1.936 & $\leftarrow$ \\
Around SR & 15.10 & 15.40 & 1.971 & $\rightarrow$ \\
Around PR & 0.69 & 0.84 & 0.923 & $\rightarrow$ \\
PR & 0.60 & 0.75 & 0.971 & $\rightarrow$ \\
Around PR & 0.45 & 0.60 & 1.020 & $\rightarrow$ \\
NR & 0.12 & 0.02 & 1.317 & $\leftarrow$ \\
\hline
\end{tabular}

region around the SR. Using NR is also possible, but sensitivity will be even lower. Therefore, PR and NR can also be used for crack detection, but they are not as advantageous as SR.

Based on the classification of resonant types, the result of crack detection using SR is presented in [16], and the results of crack detection using the other resonant types are given in $[17$, $18]$.

\section{EXPERIMENTS}

To validate the forced resonance and non-resonance currents of the probe, the calculated resonant currents were compared with the measured currents. The cutoff cavity was made with copper using electroforming technology and plated with gold, and a coaxial sliding short (Model 1909C2 at 0.8-4 GHz; Maury Microwave Corp., Ontario, CA, USA) was used as a control element. The dimensions of the cutoff cavity used in the experiments are $a=40 \mathrm{~mm}, b=20 \mathrm{~mm}, c=80 \mathrm{~mm}$, $s_{1}=30 \mathrm{~mm}, s_{2}=60 \mathrm{~mm}, t_{1}=t_{2}=a / 2$, and $r_{1}=r_{2}=$ $1 \mathrm{~mm}$. An NEC $1 \mathrm{SS} 99$ diode and ammeter (20 $\mu \mathrm{A}$ range) were connected to the parasitic post. The operating frequency used was $1 \mathrm{GHz}$, and the length of the control element was adjusted to obtain the forced resonance in the cutoff cavity.

Fig. 6 shows a photograph of the manufactured cutoff cavity probe with the control element and experimental setup. Fig. 7 shows the calculated and measured resonance currents for the ammeter connection. The calculated currents showed a reasonable agreement with the experimental results. The deviation could be due to the losses of the cutoff cavity and the control element and the effect of fabrication errors.

\section{CONCLUSION}

This study considered the forced resonance characteristics of a cutoff cavity probe that could be applied to crack detection
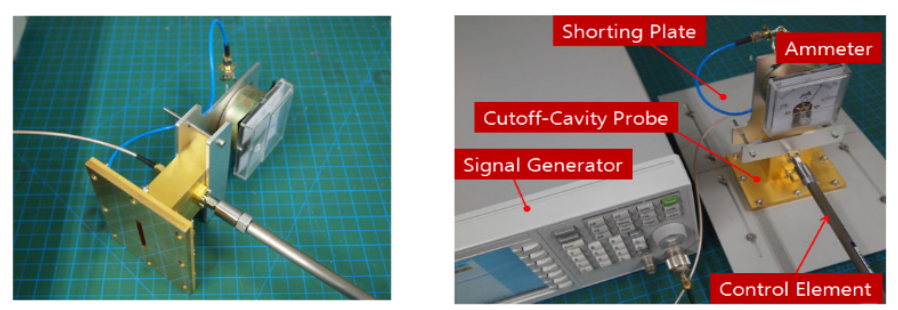

Fig. 6. Photograph of the experimental setup.

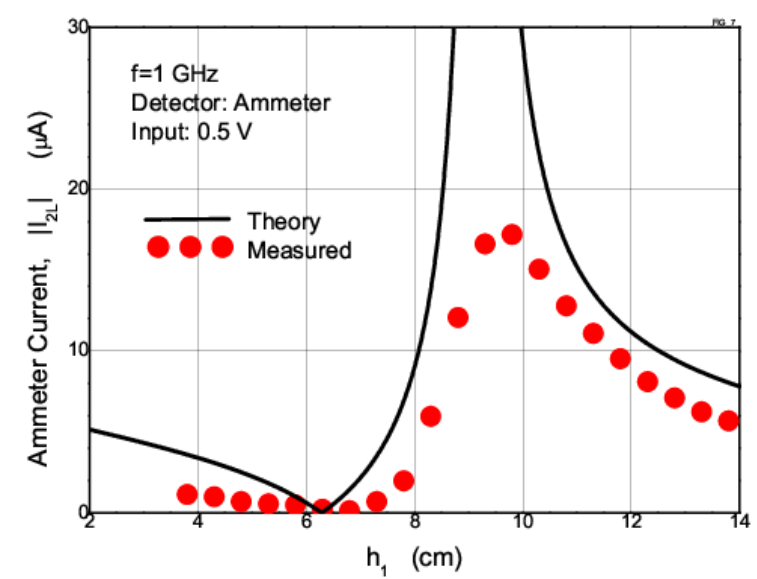

Fig. 7. Measured detector currents. 
using FRMM. There were four kinds of current states: maximum current (at the SR), minimum current (at the NR), rapidly changing current (around the SR), and slowly changing current (at the PR and around the PR). These current characteristics were classified. The SR (maximum current) and the region around SR were the most applicable current states. The NR, PR, and the region around $\mathrm{PR}$ are also available for crack detection, but their sensitivity is lower than that of SR and the surrounding region. The actual crack detection using these current states can be examined in future works.

This research was supported by the Basic Science Research Program through the National Research Foundation of Korea (NRF), which is funded by the Ministry of Education (No. NRF-2019R1D1A1A09058357).

\section{REFERENCES}

[1] K. G. Boving, NDE Handbook: Non-destructive Examination Methods for Condition Monitoring. London, UK: Butterworths, 1989.

[2] R. Zoughi, Microwave Non-destructive Testing and Evaluation. Dordrecht, The Netherlands: Kluwer Academic Publishers, 2000.

[3] A. J. Bahr, Microwave Nondestructive Testing Methods. London, UK: Gordon and Breach, 1982.

[4] C. Y. Yeh and R. Zoughi, "A novel microwave method for detection of long surface cracks in metals," IEEE Transactions on Instrumentation and Measurement, vol. 43, no. 5, pp. 719-725, 1994.

[5] C. Y. Yeh and R. Zoughi, "Microwave detection of finite surface cracks in metals using rectangular waveguide sensors," Research in Nondestructive Evaluation, vol. 6, no. 1, pp. 35-55, 1994.

[6] R. Zoughi, S. I. Ganchev, and C. Huber, "Microwave measurement-parameter optimization for detection of surface breaking hairline cracks in metals," Nondestructive Testing and Evaluation, vol. 14, no. 5, pp. 323-337, 1998.

[7] H. H. Park, Y. H. Cho, and H. J. Eom, "Surface crack detection using flanged parallel-plate waveguide," Electronics Letters, vol. 37, no. 25, pp. 1526-1527, 2001.

[8] R. Zoughi and S. Kharkovsky, "Microwave and millimetre wave sensors for crack detection," Fatigue \& Fracture of Engineering Materials \& Structures, vol. 31, no. 8, pp. 695-713, 2008.

[9] K. M. Donnell, A. McClanahan, and R. Zoughi, "On the crack characteristic signal from an open-ended coaxial probe," IEEE Transactions on Instrumentation and Measurement, vol. 63, no. 7, pp. 1877-1879, 2014.

[10] J. Kerouedan, P. Queffelec, P. Talbot, C. Quendo, S. De Blasi, and A. Le Brun, "Detection of micro-cracks on metal surfaces using near-field microwave dual-behavior resonator filters," Measurement Science and Technology, vol. 19, no. 10, article no.105701, 2008.

[11] A. Husain and E. A. Ash, "Microwave scanning microscopy for non-destructive testing," in Proceedings of 1975 5th European Microwave Conference, Hamburg, Germany, 1975, pp. 213-217.

[12] A. M. Albishi and O. M. Ramahi," Microwaves-based high sensitivity sensors for crack detection in metallic materials," IEEE Transactions on Microwave Theory and Techniques, vol. 65, no. 5, pp. 1864-1872, 2017.

[13] A. M. Albishi, M. S. Boybay, and O. M. Ramahi, "Complementary split-ring resonator for crack detection in metallic surfaces," IEEE Microwave and Wireless Components Letters, vol. 22, no. 6, pp. 330-332, 2012.

[14] T. Yun and S. Lim, "High-Q and miniaturized complementary split-ring resonator-loaded substrate integrate waveguide microwave sensor for crack detection in metallic materials," Sensors and Actuators A: Physical, vol. 214, pp. 2530, 2014.

[15] M. U. Memon and S. Lim, "Review of electromagneticbased crack sensors for metallic materials (recent research and future perspectives)," Metals, vol. 6, article no. 172, 2016.

[16] K. C. Kim, J. Y. Kwon, and N. W. Kang, "A novel forcedresonance microwave method to detect surface cracks in metal," IEICE Electronics Express, vol. 13, no. 17, article no. $715,2016$.

[17] K. C. Kim and N. W. Kang, "Crack detection system and crack detection method using the same," Korea Patents 101730872, Apr. 21, 2017.

[18] K. C. Kim, "Apparatus for conductor crack detection using electromagnetic wave and cutoff cavity probe," Korea $\mathrm{Pa}$ tents 10-2019-0171921, Dec. 2019. 


\section{Ki-Chai Kim}

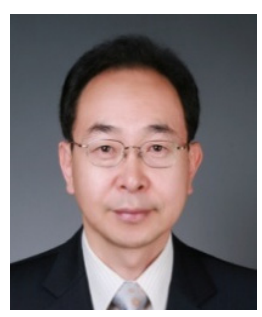

received his B.S. degree in electronic engineering from Yeungnam University, Korea, in 1984, and his M.S. and Doctor of Engineering degrees in electrical engineering from Keio University, Japan, in 1986 and 1989, respectively. Working on electromagnetic compatibility, he was a senior researcher at the Korea Standards Research Institute, Daedok Science Town, Korea, until 1993. From 1993 to 1995, he was an associate professor at Fukuoka Institute of Technology, Fukuoka, Japan. Since 1995, he has been with Yeungnam University, Gyeongsan, Korea, where he is currently a professor at the Department of Electrical Engineering. He received the 1988 Young Engineer Awards from the Institute of Electronics, Information and Communication Engineers of Japan and received a Paper Presentation Award in 1994 from The Institute of Electrical Engineers of Japan. Prof. Kim served as president of the Korea Institute of Electromagnetic Engineering and Science (KIEES) in 2012. His research interests include the EMC/EMI antenna evaluation, electromagnetic penetration problems in slots, and application of electromagnetic fields and waves.

Jong-Woo Kim

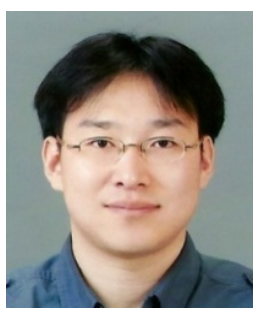

was born in Seoul, Korea. He received his B.S. and M.S. degrees in electronic engineering from Yeungnam University, Korea, in 2009 and 2012, respectively. He was an assistant manager at the reliability center of Daesung Corporation, Ansan, Korea, from 2004 to 2006, and he worked on electromagnetic compatibility. He was a team manager at the Korea Marine Equipment Research Institute (KOMERI) from 2007 to 2015 and worked in the electromagnetic compatibility test team. He is currently a center manager at KOMERI, working in the marine electronics testing and certification center.
Jae-Yong Kwon

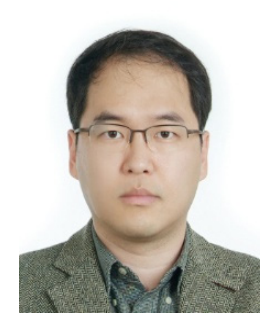

received the B.S. degree in electronics from Kyungpook National University, Daegu, in 1995, and the M.S. and Ph.D. degrees in electrical engineering from the Korea Advanced Institute of Science and Technology, Daejeon, Korea, in 1998 and 2002, respectively. He was a Visiting Scientist at the $\mathrm{Na}^{-}$ tional Institute of Standards and Technology (NIST) in Boulder, CO, USA, in 2010. From 2002 to 2005, he was a Senior Research Engineer at the Devices and Materials Laboratory, LG Electronics Institute of Technology, Seoul, Korea. He has been with the Korea Research Institute of Standards and Science, Daejeon, Korea, since 2005, where he is currently the Head of Electromagnetic Wave Metrology Group and a Principal Research Scientist. Since 2013, he has been a Professor in Science of Measurement with the University of Science and Technology, Daejeon, Korea. His current research interests include electromagnetic power, impedance, and antenna measurement. Dr. Kwon is a Korea Institute of Electromagnetic Engineering and Science (KIEES) Life Member and IEICE member.

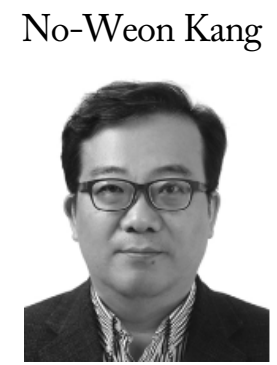

received his B.S., M.S., and Ph.D. degrees in electrical engineering from Seoul National University, Seoul, South Korea, in 1991, 1994, and 2004, respectively. His Ph.D. degree was focused on the numerical analysis of the finite-difference timedomain method, in particular, an algorithm that reconstructs the complex permittivity profile of unknown scatterers. He was with the Electromagnetic Apparatus Laboratory, LG Industrial Systems, Seoul, from 1994 to 1999, where he was involved in the static magnetic field analysis of electrical apparatus. He has been with the Korea Research Institute of Standards and Science, Daejeon, South Korea, since 2004, where he is currently the Director of the Division of Physical Metrology and a Principal Research Scientist of electromagnetic metrology. His current research interests include electromagnetic field strength, antenna measurement standards, electromagnetic interference/electromagnetic interference issues, and electro-optic sensor. 